\title{
Study of Titanium Dioxide Nanotube Array for the Application in Dye-Sensitized Solar Cells
}

\author{
Swati Bhardwaj, Tushar Rana, Pinaki Laha, Anjan Barman, and Subhayan Biswas
}

\begin{abstract}
Highly ordered, self-organized $\mathrm{TiO}_{2}$ nanotube arrays (TNA) have been successfully prepared by anodization of titanium foil in ethylene glycol electrolyte containing $0.01 \%$ ammonium fluoride (NH4F). The effect of variation of applied anodization voltage ranging from $50 \mathrm{~V}$ to $57 \mathrm{~V}$ on the morphology of the TNA has been studied using field emission scanning electron microscope. The increase in applied voltage enhances average pore size from $34 \mathrm{~nm}$ to $58 \mathrm{~nm}$ and reduces wall thickness. Diffuse reflectance spectroscopy has been used to evaluate the amount of dye absorption on the surface of various TNA which reveals direct correlation between the dye absorption and the morphology of the sample.
\end{abstract}

Index Terms-Titanium dioxide, Dye-sensitized solar cell, anodization

\section{INTRODUCTION}

In the recent years, vertically oriented, highly ordered titanium dioxide $\left(\mathrm{TiO}_{2}\right)$ nanotube array, prepared by anodization of titanium, and has attracted huge attention [1][5]. Highly ordered vertically oriented nanotube architecture of high surface to volume ratio exhibit unique properties. Nanotube offers a larger interfacial area due to their external and internal surfaces, which make them suitable for applications in dye-sensitized solar cells [6]. It also provides excellent electron pathways for charge transfer between interfaces. $\mathrm{TiO}_{2}$ nanostructures can be synthesized by various methods like sol gel transcription using organogelator template [6]-[7], seeded growth mechanism [8], hydrothermal techniques [9]. Among these processes, the electrochemical anodization of titanium in fluorinated electrolytes is a simple method to synthesize porous highly vertically ordered structures with high aspect ratios. The process of self organization of nanotube is a complex mechanism [10]-[13] and requires extensive study. The properties of self-organized TNA can be tailored using various anodization conditions and post deposition treatment. In the present work, anodization condition is varied to observe change in the morphology of TNA and the corresponding dye absorption on TNA surface. Various anodization voltages ranging from $50-57 \mathrm{~V}$ has been applied to prepare TNA samples.

Manuscript received July 5, 2013; revised September 1, 2013.

Swati Bhardwaj, Tushar Rana, and Subhayan Biswas are with the Department of Physics, the LNM Institute of Information Technology, Jaipur 302031, Rajasthan, India (e-mail: swati1783@gmail.com, tushar.18985@gmail.com, subhayanb@gmail.com).

Pinaki Laha and Anjan Barman are with the S. N. Bose National Centre for Basic Sciences, Sector-III, Block - JD, Salt Lake, Kolkata-700098, India (e-mail: pinaki@bose.res.in, abarman@bose.res.in).

\section{MATERIALS AND METHOD}

Titanium sheet $(99.9 \%$ purity, Sigma Aldrich) with a thickness of $0.25 \mathrm{~mm}$ was taken for the anodization. Small pieces of $\mathrm{Ti}^{-}$sheet of size $1 \times 1 \mathrm{~cm}$ were cleaned by sonicating separately in acetone, methanol, and finally with de-ionized water (DI), for $10 \mathrm{~min}$ each. Electrochemical anodization of titanium was carried using a two-electrode configuration with $\mathrm{Ti}$ as working electrode and $\mathrm{Pt}$ as counter electrode. The distance between the two electrodes kept at about $12 \mathrm{~cm}$. Anodization was performed in room temperature under constant-voltage conditions using a source meter (Model-2400 Keithley Instruments, Inc) and the current response of the sample was monitored in real time. All experiments were performed at room temperature with different constant anodization voltages ranging from 50 to $57 \mathrm{~V}$ for $30 \mathrm{~min}$ time duration. Ethylene glycol, ammonium fluoride and DI-water were used as electrolyte in the electrochemical anodic reaction. The electrolyte was stirred continuously during the anodization process. The anodized samples were washed with ethanol, ultra-sonicated for $10 \mathrm{~min}$ to remove the debris from the surface. Anodized samples were annealed at $450^{\circ} \mathrm{C}$ for 1 hour in air. The absorbance of Ru-based N719 dye on TNA was obtained by diffuse reflectance UV-vis spectroscopy (DRS) using integrated sphere (ISR 240A) attached with UV-vis spectrophotometer (Shimatdzu-2450) The morphology of different TNA was studied by field emission scanning electron microscope (Quanta FEG250).

\section{RESULTS AND DISCUSSIONS}

The Fig. 1 represents the anodization current vs. time for the TNA, deposited with $50 \mathrm{~V}$ and $57 \mathrm{~V}$. Initially, with the increase of voltage, anodization current increases rapidly and reaches maximum point with in $150 \mathrm{sec}$. After that an abrupt decrease of current is observed which is due to the formation of an oxide barrier layer that induces a voltagedrop between the working electrode, Ti layer, and electrolyte. In this region, electronic conduction decreases and ionic conduction through the $\mathrm{TiO}_{2}$ increases. This stage is followed by a steady state, when anodization current reaches a saturation value. At this stage dissolution and oxidation of titanium reaches a kind of equilibrium which leads to maximization of formation of nanotube. The anodization current depends upon different phenomena that take place during anodization process [14]. Higher anodization voltage enhances both field assisted movement of fluorine ion towards $\mathrm{TiO}_{2}$ layer at the electrolyte/oxide interface and ejection of $\mathrm{Ti}_{4}+$, which in turn induces more dissolution of $\mathrm{TiO}_{2}$. The Fig. 1 indicates that increase of 
anodization voltage enhances anodization current in the entire deposition period. It is important to note that the samples, deposited at $55 \mathrm{~V}$ and $57 \mathrm{~V}$, show comparatively high anodization current even when it reaches steady state conditions.

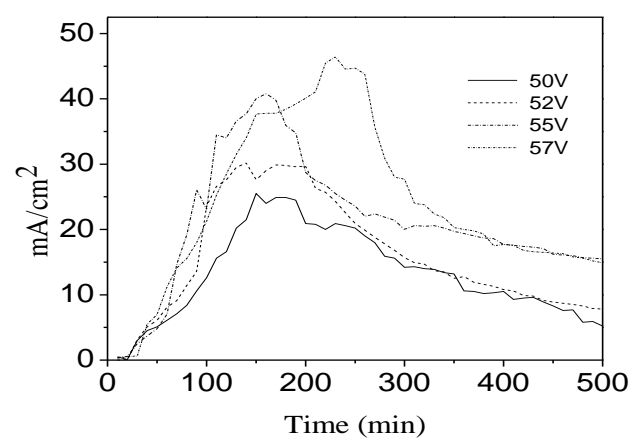

Fig. 1. Anodization current vs. time of $\mathrm{TiO} 2$ nanotubes, deposited at various anodization voltages.

Fig. 2 shows a comparison of XRD patterns of nanotubes samples after their formation and after annealing at $450^{\circ} \mathrm{C}$ in air. The X-ray diffraction measurement reveals that although as-deposited film shows amorphous nature but annealed film shows the anatase peaks (101), (211) and (216).

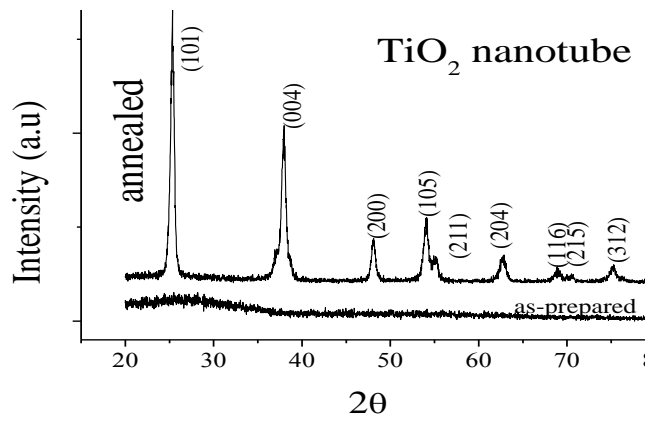

Fig. 2. X-ray diffraction of $\mathrm{TiO} 2$ nanotube array: as-prepared and annealed

The field emission electron microscopy (FESEM) images of the nanotubes deposited at different potentials from $50 \mathrm{~V}$ to $57 \mathrm{~V}$ are shown in Fig. 3. Gradual change has been observed in the diameter of the pore and wall-thickness of $\mathrm{TiO}_{2}$ nanotube as anodization voltage is increased from $50 \mathrm{~V}$ to $57 \mathrm{~V}$. For TNA, prepared with $57 \mathrm{~V}$, etching of top layer leads to very low wall thickness. The distribution of porediameter is shown in Fig. 4, which reveal definite increase of pore diameter. The average pore diameter of the $\mathrm{TiO}_{2}$ nanotube prepared at $50,52,55$ and $57 \mathrm{~V}$ is $34 \pm 4,44 \pm 3$, $53 \pm 5$ and $58 \pm 4 \mathrm{~nm}$ respectively. Fig. 5 is an indicative figure, which shows highly ordered vertically arranged $\mathrm{TiO}_{2}$. nanotube prepared at 52V. Fig. 6 presents UV-vis diffuse reflectance spectra of $\mathrm{TiO}_{2}$ nanotube prepared at different anodization voltages. The entire sample show typical variation of diffuse reflectance of $\mathrm{TiO}_{2}$ nanotube array [15]. The reflectance of $\mathrm{TiO}_{2}$ nanotube array prepared on $\mathrm{Ti}$ metal, increases with increase of anodization voltage. This is due to the voltages. The possible reason behind the variation of variation of thickness of TNA, prepared at different thickness with anodization voltage is that increase of anodization voltage enhances anodization current substantially, as shown in Fig. 1. This high current enhances temperature of the electrolyte which in turn reduces the deposition rate and thickness [16].

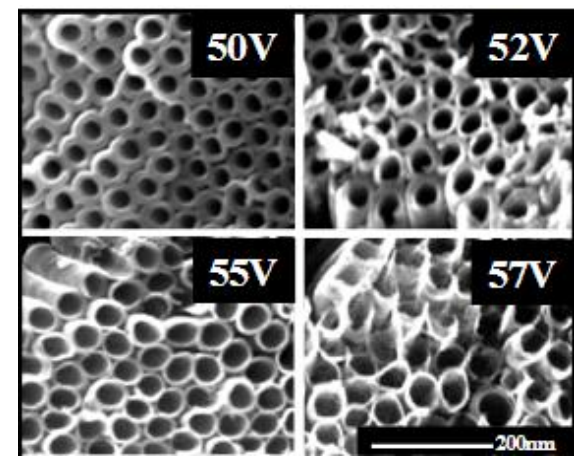

Fig. 3. FESEM images of top view of TiO2 nanotube array, deposited at anodization voltage $50 \mathrm{~V}, 52 \mathrm{~V}, 55 \mathrm{~V}$ and $57 \mathrm{~V}$.

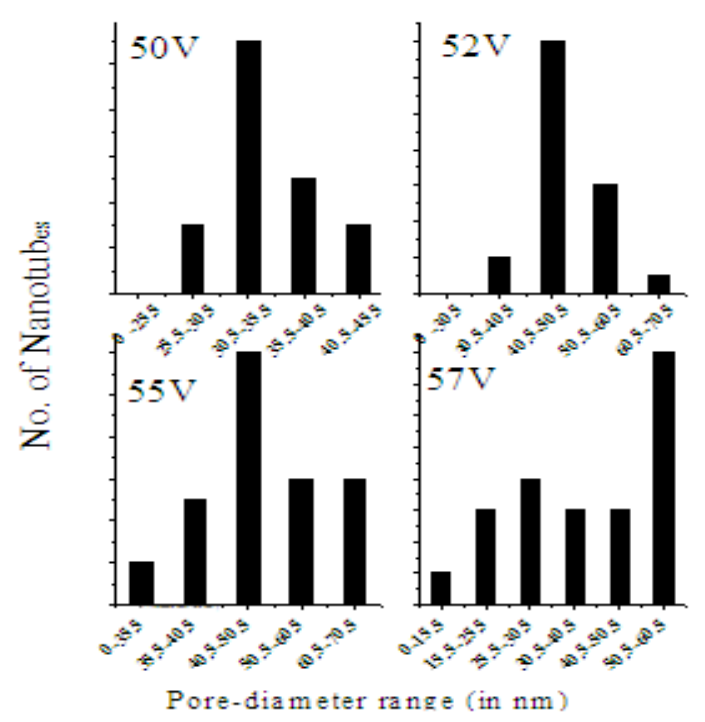

Fig. 4. Pore size (nm) distribution of $\mathrm{TiO}_{2}$ nanotube array, deposited at anodization voltage $50 \mathrm{~V}, 52 \mathrm{~V}, 55 \mathrm{~V}$ and $57 \mathrm{~V}$.

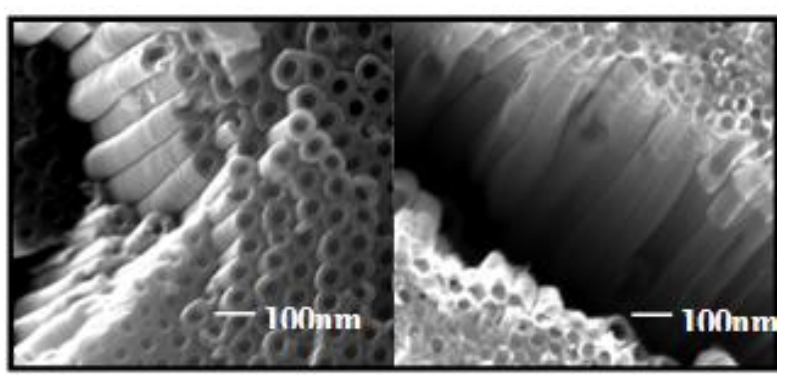

Fig. 5. Side view of $\mathrm{TiO}_{2}$ nanotube array prepared with $52 \mathrm{~V}$ anodization voltage.

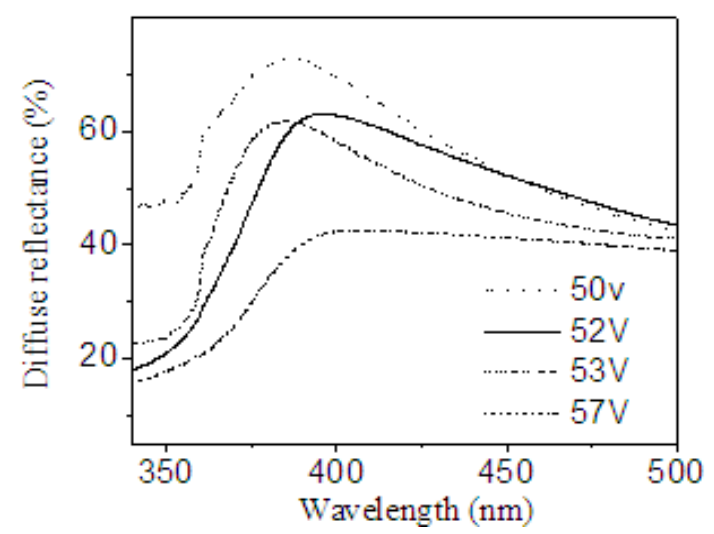

Fig. 6. Diffuse reflection spectra of $\mathrm{TiO}_{2}$ nanotube array, prepared at different anodization voltages.

The measured reflectance was converted directly to 
absorbance $(\log (1 / R))$ where $R$ represents relative diffuse reflectance. Since the purpose of the reflectance measurements was not to determine the N719 concentration but to measure the absorbance of the N719 adsorbed on TNA, the Kubelka-Munk function, was not used [17]. Fig. 7 presents variation of absorbance of $\mathrm{TiO}_{2}$ nanotube array, prepared at 50 and $57 \mathrm{~V}$ (with and without dye), with wavelength. It has been observed that in the visible light range, dye-sensitized TNA, deposited at 50V exhibits much lesser enhancement of absorbance, in compare to the sample, deposited with $57 \mathrm{~V}$. It is due to the fact that increase of pore diameter and decrease of wall thickness of TNA, deposited at higher anodization voltage, provide more surface area for dye-absorption.

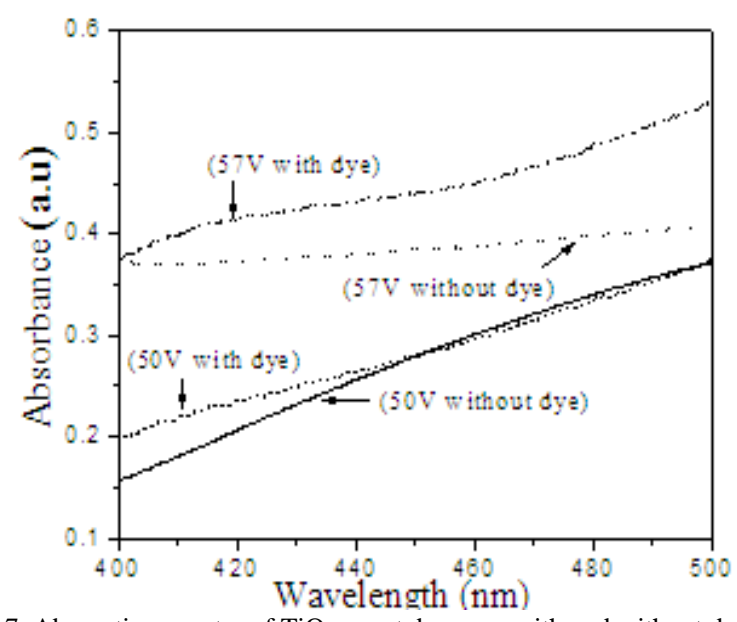

Fig. 7. Absorption spectra of $\mathrm{TiO}_{2}$ nanotube array with and without dye, prepared at 50 and $57 \mathrm{~V}$.

\section{CONCLUSION}

In conclusion, vertically ordered $\mathrm{TiO}_{2}$ nanotubes array have been successfully prepared by anodization technique with varying anodization voltages ranging from $50 \mathrm{~V}$ to $57 \mathrm{~V}$ to study the variation of dye absorption. The study reveals that increase of anodization voltage induces systematic changes in the morphology of TNA. This gradual morphological change is very useful for fine tuning properties that are appropriate for various applications. The effect of increase of applied anodization voltage has been reflected in the real time measurement of anodization current. In this present investigation, FESEM measurement confirms that increase of anodization voltage enhances diameter of the $\mathrm{TiO}_{2}$ nanotubes and reduces wall thickness which provide higher surface area for dye-absorption. The absorbance, measured by diffuse reflectance spectroscopy, shows maximum values for N719 sensitized TNA, prepared at $57 \mathrm{~V}$.

\section{ACKNOWLEDGMENT}

This research is supported by funding from LNMInstitute of Information Technology, Jaipur.

\section{REFERENCES}

[1] C. A. Grimes and G. K. Mor., $\mathrm{TiO}_{2}$ Nanotube Arrays: Synthesis, Properties, and Applications, Springer, 2009.

[2] K. Shankar, J. I. Basham, N. K. Allam, O. K. Varghese, G. K Mor., X Feng., M. Paulose, J. A. Seabold, K. S. Choi, and C. A. Grimes "Recent advances in the use of $\mathrm{TiO}_{2}$ nanotube and nanowire arrays for oxidative photoelectrochemistry," J. Phys. Chem. C, vol. 113, pp. 6327-6359, March 2009.

[3] J. M. Macak, H. Tsuchiya, A. Ghicov, K. Yasuda, R. Hahn, S. Bauer, and P. Schmuki, " $\mathrm{TiO}_{2}$ nanotubes: self-organized electrochemical formation, properties and applications," Solid State and Material Science, vol. 11, pp. 3-18, Aug. 2007.

[4] G. K. Mor., O. K. Varghese, M. Paulose, K. Shankar, and C. Grimes, "Highly ordered and vertically oriented $\mathrm{TiO}_{2}$ nanotube array: fabrication and material properties," Solar Energy Materials \& Solar Cells, vol. 90, pp. 2011-2075, Sept. 2006.

[5] F. A. Nada, M. A. Haten, M. Amin, W. Kalil, and A. Galal, "Nanotube arrays as photoanodes for Dye sensitized solar cells using mctal phthaocyanine," Int. J. Electrochem. Sci., vol. 6, pp. 33163332, Aug. 2011.

[6] A. N. Banerjee et al. "The design, fabrication, and photocatalytic utility of nanostructured semiconductors: focus on $\mathrm{TiO}_{2}$-based nanostructures," Nanotechnology, Science and Applications, vol. 4, pp. 35-65, Feb. 2011.

[7] S. Kobayanshi, N. Hamasaki, M. Sizuki, M. Kimura, H. Shirai, and K. Hanabusa, "Preparation of helical transition-metal oxide tubes using organogelators as structure-directing agents," J. Am. Chem. Soc., vol. 124, pp.6550-6551, May 2002.

[8] Z. R. R. Tian, J. A. Voigt, J. Liu, B. Mckenzie, and H. F. Xu, "Large oriented arrays and continuous films of $\mathrm{TiO}_{2}$-based nanotubes," $J$. Am. Chem. Soc., vol. 125, pp.12384-12385, Sept. 2003.

[9] T. Kasuga, M. Hirasamastu, A. Hoson, T. Sekino, and K. Nikahara, "Formation of titanium oxide nanotube," Langmuir, vol. 14, pp. 3160 -3163 , May 1998.

[10] Z. X. Su, W. Z. Zhou, "Formation, morphology control and applications of anodic $\mathrm{TiO}_{2}$ nanotube arrays," J. Mater. Chem., vol. 21, pp.8955-8970, April 2011.

[11] A. Ghicov and P. Schmuki, "Self-ordering electrochemistry: a review on growth and functionality of $\mathrm{TiO}_{2}$ nanotubes and other self-aligned $\mathrm{MO}_{x}$ structures," Chem. Commun., vol. 20, pp. 2791-2808, April 2009

[12] G. Adrian, R. Morales, O. María, C. Guzmán, and C. C. Arteaga, "A brief review on fabrication and applications of auto-organized $\mathrm{TiO}_{2}$ nanotube arrays," Corrosion Review, vol. 29, pp. 105-121, Sept. 2011

[13] V. R. Subramanian, S. Sarker, B. W. Yu, A. Kar, X. D. Sun, and K. D. Sandwip, " $\mathrm{TiO}_{2}$ nanotubes and its composites: Photocatalytic and other photo-driven applications," J. Mat. Res, vol. 28, pp. 280-293, Jan. 2013.

[14] Y. S. Jun, J. H. Park, and M. G. Kang, "The preparation of highly ordered $\mathrm{TiO}_{2}$ nanotube arrays by an anodization method and their applications, " Chem. Commun., vol. 48, pp. 6456-6471, April 2012.

[15] B. X. Lei, J. Y. Liao, R. Zhang, J. Wang, C. Y. Su, D. B. Kuang, "Ordered crystalline $\mathrm{TiO}_{2}$ nanotube arrays on transparent FTO glass for efficient dye-Sensitized Solar Cells," J. Phys. Chem C, vol. 114 pp.15228, Aug. 2010.

[16] H. E. Prakasam, K. Shankar, M. Paulose, O. K. Varghese, and C. A Grimes, "A new benchmark for $\mathrm{TiO}_{2}$ nanotube array growth by anodization," Journal of Physical Chemistry C, vol. 111, no. 20, pp 7235-7241, April 2007.

[17] W. Wendlandt and H. Hecht, Reflectance Spectroscopy, Interscience Publishers, John Wiley \& Sons, New York, vol. 21, 1966.

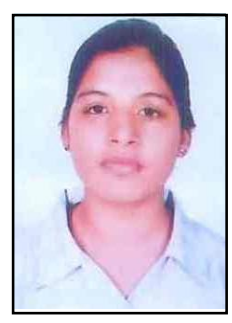

Swati Bhardwaj was born in Hardwar, Uttar Pradesh, India in 1983. She did M.Sc. in Physics from Gurukhul Kangri Vishwavidyalaya, Hardwar, India in 2007. Her current research interests are nanostructured semiconductors and third generation photovolatic device.

Presently she is a Ph.D. student at The LNM Institute of Information Technology, Jaipur, India.

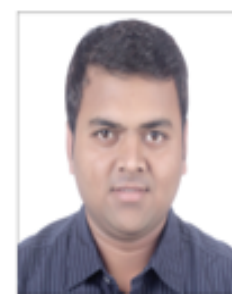

Tushar Rana was born in Sojitra, Gujarat, India in 1985. He did M.Sc. in Material Science (Nanotechnology) from The Maharaja Saajirao University Vadodara, Gujrat, India in 2010. His current research interests are nano-structural materials synthesis and theoretical condensed matter physics.

Presently he is a Ph.D. student at The LNM Institute of Information Technology, Jaipur, India. 


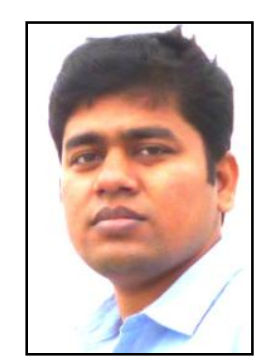

Pinaki Laha was born in Midnapore, West Bengal, India in 1984. He did M.Sc. in Physics from Vidyasagar University, West Bengal, and Ph.D. from Birla Institute of Technology, Mesra, Ranchi, India. His current research interests are thin film magnetism, nanostructure, plasma spectroscopy.

Presently $\mathrm{He}$ is a post doctoral research associate in S. N. Bose National Centre for Basic Sciences, Kolkata, India.

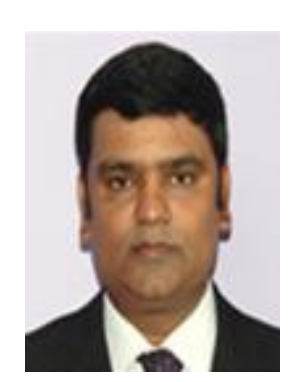

Anjan Barman was born in West Bengal, India in 1969. He did Ph.D. in Physics from Indian association for the Cultivation of Science, India in 1999. His current research interests are nanomagnetism, spintronics, ultrafast laser spectroscopy, high frequency dynamics, magnonics.
He was an assistant professor at Indian Institute of Technology, Delhi. Presently he is an associate professor at the S. N. Bose National Centre for Basic Sciences, Kolkata, India.

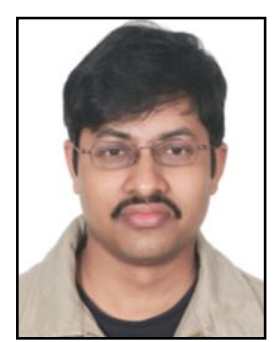

Subhayan Bis was was born in Santiniketan, West-Bengal, India in 1972. He did Ph.D. from Indian association for the Cultivation of Science, India in 2003. His current research interest is nanostructured semiconductor and third generation photovoltaic devices.

He did postdoctoral work at Inha University, South Korea, National Sun Yat-Sen University, Taiwan, National Taiwan University, Taiwan and University of Toyama, Japan. Presently he is an Information Technology, Jaipur, India. 